\title{
Leptonic and semileptonic decays with taus at the Belle II experiment
}

\section{Marco Milesi $^{a, *}$ on behalf of the Belle II Collaboration}

${ }^{a}$ School of Physics, The University of Melbourne,

Parkville 3010 Victoria, Australia

E-mail: marco.milesi@unimelb.edu.au

Lepton flavour universality as predicted by the Standard Model is challenged by various measurements of $B$ meson decays involving tau and charged light leptons. The Belle II experiment started recording first collision data in spring 2018 and offers a unique laboratory to study lepton flavour universality in leptonic and semileptonic $B$ decays at high precision. Here we report the status of establishing first measurements of (semi) tauonic $B$ decays and preliminary results of the $B \rightarrow \tau \bar{v}_{\tau}$ channel using the available Belle II data, and discuss the future potential of investigating $R\left(D^{(*)}\right)$ anomalies.

40th International Conference on High Energy physics - ICHEP2020

July 28 - August 6, 2020

Prague, Czech Republic (virtual meeting)

${ }^{*}$ Speaker 


\section{Introduction}

Semileptonic $B$ decays with tau leptons $B \rightarrow(X) \tau \bar{v}_{\tau}$, where $X$ is one or more hadrons, are excellent channels for testing lepton flavour universality violation at $B$-factories, owing to the large mass of the tau. Currently, anomalies at the level of $3 \sigma$ are observed in the measurement of the ratio $R\left(D^{(*)}\right)=\mathcal{B}\left(B \rightarrow D^{(*)} \tau v_{\tau}\right) / \mathcal{B}\left(B \rightarrow D^{(*)} \ell v_{\ell}\right)$ [1]. High precision measurements of $B$ semitauonic decays in Belle II will provide a way to ultimately test any significant deviation from the Standard Model (SM), which might be explained by two-Higgs doublet models [2], or leptoquark models [3].

Furthermore, pure leptonic $B \rightarrow \tau \bar{v}_{\tau}$ decays are also of high interest, as they can provide unique sensitivity to new phenomena and a complementary measurement of the CKM matrix element $V_{u b}$ to the semileptonic channels with charged light leptons $(e, \mu)$.

In this work, we describe the analysis strategy defined for these measurements, with preliminary results on the data-driven calibration of electron and muon identification algorithms, and a review of the first Belle II results on the analysis of $B \rightarrow \tau \bar{v}_{\tau}$ to demonstrate its readiness towards analysis of a larger dataset.

\section{The Belle II experiment}

The Belle II experiment [4] is a at the SuperKEKB [5] asymmetric $e^{+} e^{-}$collider in Tsukuba, Japan. It is designed to cover a broad research programme in the flavour physics sector, including searches for New Physics in rare $B$ meson decays and high precision measurements of SM parameters. Owing to a smaller beam profile and higher current, the design SuperKEKB luminosity is $8 \times 10^{35} \mathrm{~cm}^{-2} \mathrm{~s}^{-1}$, about 40 times larger than that of its predecessor, KEKB. By the end of its lifetime, Belle II will accumulate an integrated luminosity of around $50 \mathrm{ab}^{-1}$, a dataset 50 times larger than the one of the Belle experiment. At the time of writing, Belle II has recorded $64 \mathrm{fb}^{-1}$ of data at the $\Upsilon(4 S)$ resonance, of which $34.6 \mathrm{fb}^{-1}$ have been fully processed for physics analysis and represent the dataset hereby considered.

\section{Event reconstruction strategy}

Belle II analyses of (semi) tauonic channels exploit tagging, where one of the two $B$ mesons from the $\Upsilon(4 S)$ decay is reconstructed to provide flavour and kinematic constraints on the other signal $B$ meson. Tagging in Belle II is performed by means of an algorithm named Full Event Interpretation (FEI) [6]. The FEI algorithm reconstructs $O(10,000)$ possible $B$ decay chains from the observed tracks and calorimeter clusters, by hierarchically combining intermediate states, exploiting boosted decision trees to identify at each level of the chain the most likely combination. Eventually, the algorithm provides a probability for a reconstructed $B$ to be consistent with a given decay class: a semileptonic $B$ decay, or a fully hadronic one. The $B$ tag identification efficiency (fraction of total $\Upsilon(4 S)$ candidates with a correct tag) as a function of tag purity (fraction of tagged $\Upsilon(4 S)$ candidates with a correct tag), calibrated using $B \rightarrow X \ell v$ candidates selected in data, is shown in Figure 1.

All remaining tracks and calorimeter clusters that are not used for the $B_{\text {tag }}$ reconstruction account for the remaining $B$ meson $\left(B_{\text {sig }}\right)$. Most often, the $B_{\text {sig }}$ decaying into a tau lepton is 


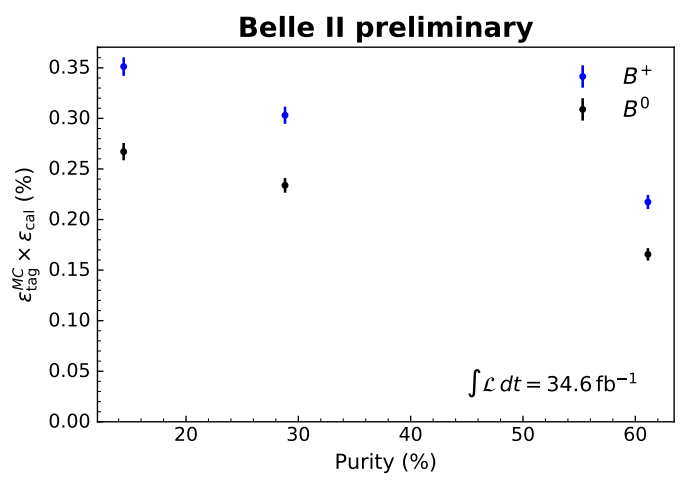

Figure 1: Tag-side identification efficiency for charged and neutral $B$ mesons in simulation against purity, corrected by the data-driven calibration correction $\varepsilon_{c a l}=N_{\text {data }}^{X \ell v} / N_{M C}^{X \ell v}$ derived from $B \rightarrow X \ell v$ events.

reconstructed through leptonic decays $\left(\mathcal{B}\left(\tau \rightarrow \ell \bar{v}_{\ell} \nu_{\tau}\right) \approx 34 \%\right)$ in order to reduce the very large hadronic background. The main variables used for signal extraction are:

- $M_{\text {miss }}^{2}=\left(p_{e^{+} e^{-}}-p_{B_{t a g}}-p_{B_{s i g}}\right)^{2}$ : invariant mass squared of the missing four-momentum. As leptonic tau decays produce two extra neutrinos, it is used to separate the signal (Figure 2a) from $B \rightarrow X \ell v$ events (Figure $2 b$ ) as well as purely hadronic final states.

- $E_{E C L}$ : energy in the electromagnetic calorimeter (ECL) of neutral particles not used in the reconstruction of the signal or tag $B$. Well reconstructed signal candidates are expected to have little to no $E_{E C L}$ (Figure 2c).

- $p_{\ell}^{*}$ : the momentum of the lepton in the $B_{\text {sig }}$ rest frame. Leptons from tau decays are characterised by a momentum spectrum that peaks at only a few hundred $\mathrm{MeV}$ (Figure 2d), making their identification particularly challenging.

\section{Lepton identification performance in 2020 data}

Information from each particle identification system (central drift chamber, Cherenkov detectors, electromagnetic calorimeter, $K_{\mathrm{L}}^{0}$-muon detector) is analysed independently to determine a likelihood for each charged particle hypothesis. These likelihoods may then be used to construct a combined global likelihood ratio discriminator, defined for leptons $(\ell=\{e, \mu\})$ as:

$$
\ell_{\mathrm{ID}}=\frac{\mathcal{L}_{\ell}}{\mathcal{L}_{e}+\mathcal{L}_{\mu}+\mathcal{L}_{\pi}+\mathcal{L}_{K}+\mathcal{L}_{p}+\mathcal{L}_{d}} .
$$

Performance of lepton identification efficiency and hadron $(h=\{\pi, K\})$ mis-identification probability for several reference selection thresholds on the $\ell_{\mathrm{ID}}$ variable is calibrated in simulation to events selected in collision data across several standard processes, that cover a broad range of lepton candidate lab-frame momenta, from approximately $0.4 \mathrm{GeV} / c$ to $7.0 \mathrm{GeV} / c$ [7]. The polar angle range considered corresponds to the calorimeter's acceptance for electrons ( 0.22 to $2.71 \mathrm{rad})$, 


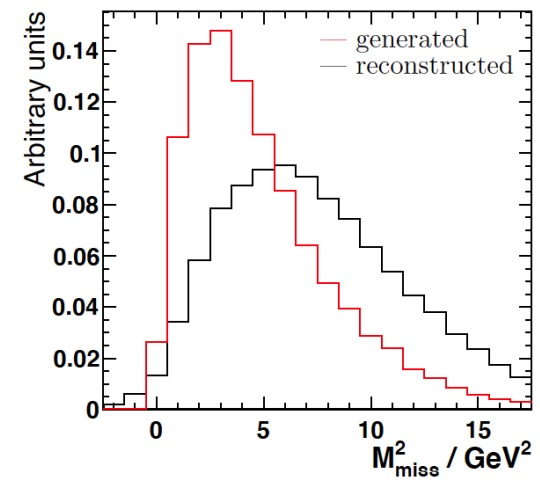

(a) $M_{\text {miss }}^{2}$ for generated (red) and fully simulated (black) signal $B \rightarrow X \tau \bar{v}_{\tau}$ events. From Belle official simulation.

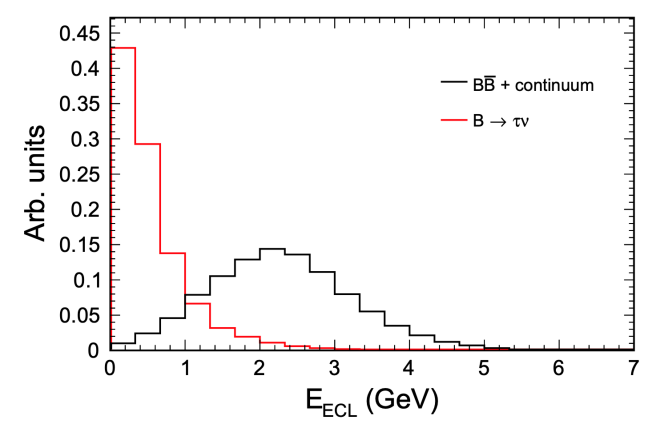

(c) $E_{E C L}$ for fully simulated signal $B \rightarrow X \tau \bar{v}_{\tau}$ (red) and background (black) events. From Belle II official simulation.

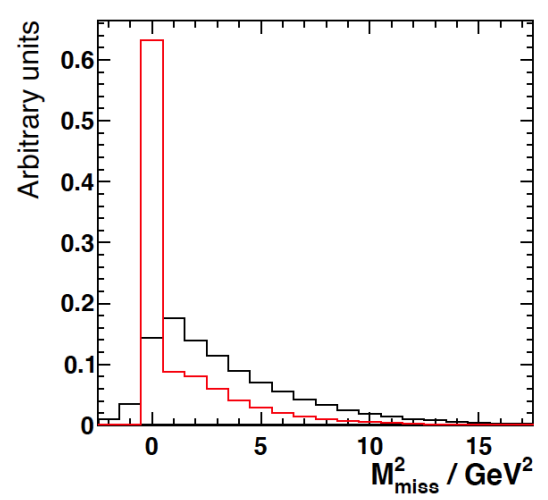

(b) $M_{\text {miss }}^{2}$ for generated (red) and fully simulated (black) signal $B \rightarrow X \ell v$ events. From Belle official simulation.

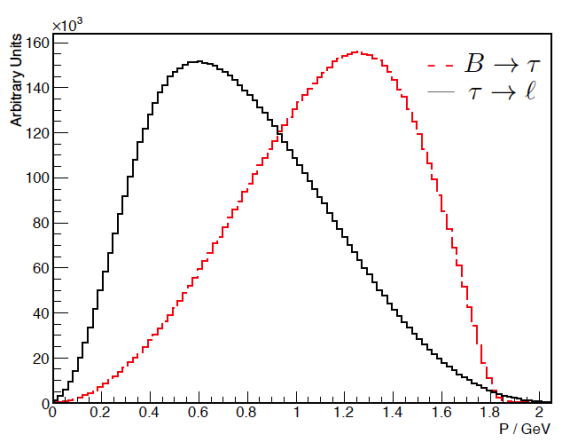

(d) $p^{*}$ distribution at generator level for $B \rightarrow X \tau \bar{v}_{\tau}$ events: the signal $B$ momentum (red) and the lepton momentum $p_{\ell}^{*}$ (black) from the $\tau \rightarrow \ell \bar{v}_{\ell} \nu_{\tau}$ decay. From Belle official simulation.

Figure 2: Monte-Carlo based distributions of variables used for signal extraction in (semi) tauonic $B$ analyses in Belle and Belle II .

and to the $K_{\mathrm{L}}^{0}$-muon detector (KLM) acceptance for muons (0.40 to $\left.2.60 \mathrm{rad}\right)$. All the channels considered are listed in Table 1, along with the probed momentum range.

Efficiencies and mis-identification probabilities for electrons and muons measured in data as a function of $p_{l a b}$ in the various channels are overlaid in Figure 3, for a threshold of $\ell_{\mathrm{ID}}>0.9$, in two representative bins of polar angle in the central "barrel" detector region. On average, we achieve an efficiency of $94 \%$ for $e$ identification (90\% for $\mu$ identification) with a $2 \% \pi$-e mis-identification probability ( $4 \%$ for $\pi-\mu$ ), respectively.

Further improvement of lepton identification performance at low momenta will be attained with a new boosted decision tree-based algorithm that combines sub-detector likelihoods with several calorimeter observables describing lateral electromagnetic shower development and pulse shape analysis from the ECL crystals' waveform readout [8]. This algorithm has been recently introduced in the Belle II analysis software, and will become the standard for lepton identification. 


\begin{tabular}{lcc}
\hline Channel & Probe particle & Approximate $p_{\text {lab }}$ range $[\mathrm{GeV} / c]$ \\
\hline \multicolumn{2}{l}{ Lepton identification efficiency } & \\
\hline$e^{+} e^{-} \rightarrow \ell^{+} \ell^{-}(\gamma)$ & $e, \mu$ & $0.4(0.7)-7.0$ \\
$e^{+} e^{-} \rightarrow e^{+} e^{-} \ell^{+} \ell^{-}$ & $e, \mu$ & $0.4-3.0$ \\
$J / \psi \rightarrow \ell^{+} \ell^{-}$ & $e, \mu$ & $1.0-3.0$ \\
\hline \multicolumn{2}{l}{ Hadron mis-identification probability } \\
\hline$e^{+} e^{-} \rightarrow \tau^{ \pm}(1 P) \tau^{\mp}(3 P)$ & $\pi$ & $0.4-4.0$ \\
$K_{S}^{0} \rightarrow \pi^{+} \pi^{-}$ & $\pi$ & $0.4-3.5$ \\
$D^{*+} \rightarrow D^{0}\left(K^{-} \pi^{+}\right) \pi^{+}$ & $\pi, K$ & $0.4-3.5$ \\
\hline
\end{tabular}

Table 1: The channels used for calibrating lepton identification efficiency and hadron mis-identification probability, along with the associated momentum range sensitivity.

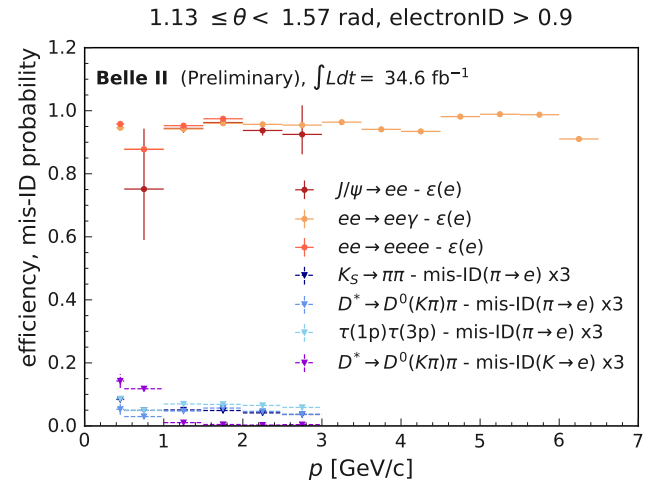

(a) Electron identification.

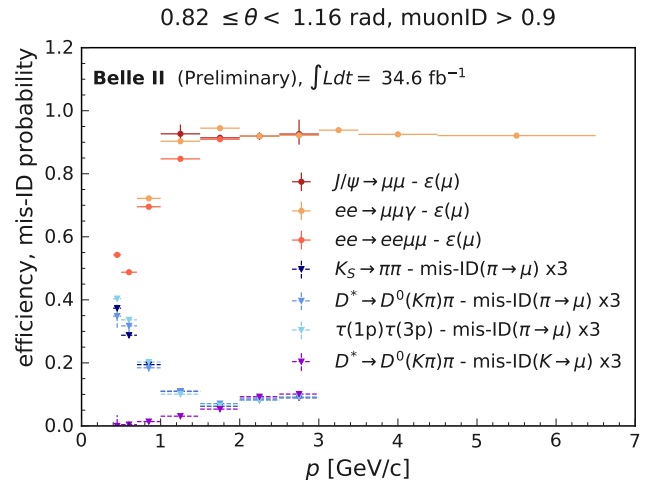

(b) Muon identification.

Figure 3: Efficiencies and mis-identification probabilities for a $\ell_{\mathrm{ID}}>0.9$ cut for electrons and muons as a function of $p_{l a b}$ for selected polar angle bins, overlaying results of several channels. Note that the mis-identification rate has been inflated by a factor 3 for illustration purposes.

\section{Preliminary results on $B \rightarrow \tau\left(\rightarrow e \bar{v}_{e} v_{\tau}\right) \bar{v}_{\tau}$}

Although a dataset size of $O\left(1 \mathrm{ab}^{-1}\right)$ will be required to establish an observation of $B \rightarrow \tau \bar{v}_{\tau}$, a preliminary study of the modelling of variables for signal extraction in this channel has been performed with the available data to test the event reconstruction capability [9].

Only the channel with final state electrons, $\tau \rightarrow e \bar{v}_{e} v_{\tau}$, is considered at this stage. The analysis exploits the FEI algorithm to tag hadronic $B$ decays on one side of the $\Upsilon(4 S)$ decay. A purity of around $50 \%$ for correctly reconstructed $B_{\text {tag }}$ candidates is obtained, as extracted from a fit to the beam-constrained mass of the $B_{\text {tag }}$ system, $M_{b c}=\sqrt{\left(E_{\text {beam }}^{*} / 2\right)^{2}-p_{B_{\text {tag }}}^{*}{ }^{2}}$ (where quantities are defined in the $B \bar{B}$ centre-of-mass reference frame).

After the $B_{\text {tag }}$ selection, a requirement of a single charged track with $p_{\text {lab }}>0.5 \mathrm{GeV} / c$ and $\mathrm{e}_{\mathrm{ID}}>0.9$ is enforced on the signal side. The Monte-Carlo simulation description of data is finally checked for the residual calorimeter energy $E_{E C L}$ neither associated to $B_{t a g}$, nor to $B_{\text {sig }}$ (Figure 4a), 
and for the sum of missing energy and missing momentum $E_{m i s s}^{*}+c p_{m i s s}^{*}$ (Figure $4 \mathrm{~b}$, the latter calculated in the $B \bar{B}$ centre-of-mass frame). For both variables, the simulated background processes in the regions where most signal is expected is found to agree with collision data.

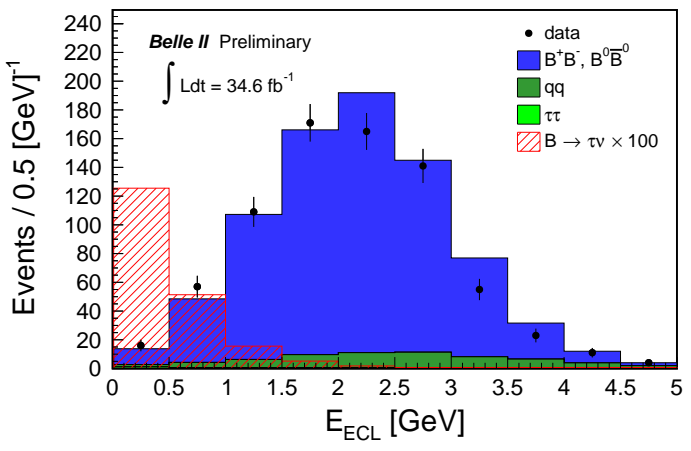

(a) $E_{E C L}$

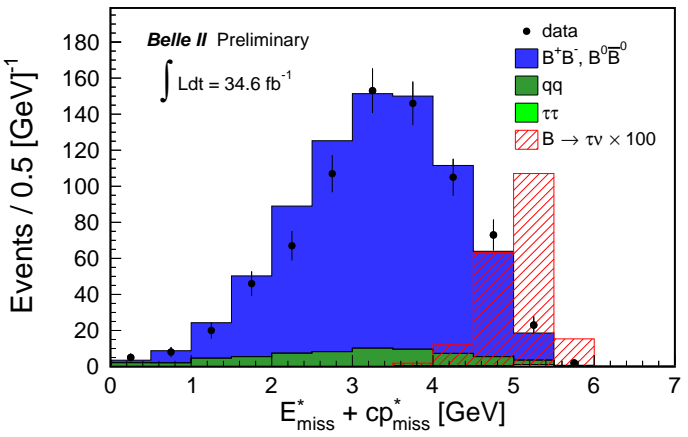

(b) $E_{m i s s}^{*}+c p_{m i s s}^{*}$

Figure 4: Distribution of the residual energy in the ECL and missing energy plus momentum for $B \rightarrow \tau(\rightarrow$ $\left.e \bar{v}_{e} v_{\tau}\right) \bar{v}_{\tau}$. The Monte-Carlo simulation has been scaled to match the collision data integrated luminosity. Note that the $B \rightarrow \tau \bar{v}_{\tau}$ signal has been enhanced by a factor 100 .

\section{Prospects for measurements of leptonic and semileptonic $B$ decays with $\tau$ leptons at Belle II}

Semitauonic decays that proceed via tree-level $b \rightarrow c \tau \bar{v}_{\tau}$ transition, such as $B \rightarrow D^{(*)} \tau \bar{v}_{\tau}$, provide a rich phenomenology to test for beyond-SM effects [10]. The well-known measurement of the $R\left(D^{(*)}\right)$ ratio by Belle II is expected to reach the precision of the current world average with $O\left(1 \mathrm{ab}^{-1}\right)$ of data, and it will already become competitive with a few hundred $\mathrm{fb}^{-1}$, namely the expected Belle II dataset size in 2021. Further improvements can be achieved by reducing the large systematic uncertainty on the branching ratio of backgrounds from excited charmed mesons decays, $B \rightarrow D^{* *} \ell \bar{v}_{\ell}$. This requires dedicated high precision measurements for this decay channel. It is estimated that with the full $50 \mathrm{ab}^{-1}$ Belle II dataset, the experimental precision on $R\left(D^{(*)}\right)$ will be comparable to the current SM theoretical uncertainty, which is $O(1 \%)$.

With a dataset size $O\left(100 \mathrm{fb}^{-1}\right)$, a novel measurement of the branching ratio of inclusive $B \rightarrow X \tau \bar{v}_{\tau}$ decays can also be performed. This analysis provides several experimental challenges, from the aforementioned identification of low momentum leptons to suppress background from misidentified hadrons, to the handling of irreducible background from cascade decays $B \rightarrow D \rightarrow \ell$. A very precise measurement of the branching ratios of simulated background processes will be paramount.

Other interesting measurements are the polarisation of the $\tau$ lepton and of the $D^{*}$ meson and the differential distribution of momentum transfer to the $\tau-\bar{v}_{\tau}$ pair, $q^{2}$, which are both sensitive to New Physics effects, although a dataset $O\left(10 \mathrm{ab}^{-1}\right)$ would be required to attain sufficient precision. 


\section{Conclusions}

Preliminary Belle II results on $B$ decays with tau leptons with $34.6 \mathrm{fb}^{-1}$ of collision data provide a successful test of improved techniques for event reconstruction, such as the FEI, and demonstrate readiness for analysis of a larger dataset in the years to come. An extensive set of calibration channels have been established to assess the performance of lepton identification over a broad momentum range. Algorithmic improvements to enhance the lepton-hadron separation at low momenta - crucial to suppress backgrounds in final states with leptonically decaying $\tau$ 's - are currently well underway, and will be exploited in the next round of physics analyses.

\section{References}

[1] Heavy Flavor Averaging Group, Averages of b-hadron, c-hadron, and $\tau$-lepton properties as of 2018, 1909.12524 (2019)

[2] G.C. Branco et al., Theory and phenomenology of two-Higgs-doublet models, 10.1016/j.physrep.2012.02.002 (2012)

[3] I. Doršner et al., Physics of leptoquarks in precision experiments and at particle colliders, 10.1016/j.physrep.2016.06.001 (2016)

[4] T. Abe et al., Belle II Technical Design Report, arXiv:1011.0352 (2010).

[5] K. Akai, K. Furukawa, H. Koiso, SuperKEKB Collider, Nucl. Instrum. Meth., A907, 188-199 (2018).

[6] T. Keck, The Full Event Interpretation, Computing and Software for Big Science, 10.1007/s41781-019-0021-8 (2019)

[7] The Belle II collaboration, Muon and electron identification efficiencies and hadron-lepton mis-identification probabilities, BELLE2-NOTE-PL-2020-027 (2020)

[8] M. Milesi et al., Lepton identification in Belle II using observables from the electromagnetic calorimeter and precision trackers, EPJ Web Conf., 10.1051/epjconf/202024506023 (2020)

[9] The Belle II collaboration, Approved plots for $B \rightarrow \tau \bar{v}_{\tau}$ study with $34.6 \mathrm{fb}^{-1}$ of Phase III data, BELLE2-NOTE-PL-2020-023 (2020).

[10] E. Kou, P. Urquijo, The Belle II Physics Book, Progress of Th. and Exp. Phys. (PTEP) 12 (2019). 